\title{
BMJ Open Electroacupuncture as an adjunctive therapy for motor dysfunction in acute stroke survivors: a systematic review and meta-analyses
}

Jie Zhan, ${ }^{1,2}$ Ruihuan Pan, ${ }^{3}$ Mingchao Zhou, ${ }^{1}$ Feng Tan, ${ }^{1,2}$ Zhen Huang, ${ }^{2}$ Jing Dong, ${ }^{1}$ Zehuai Wen ${ }^{4,5}$

To cite: Zhan J, Pan R, Zhou M, et al. Electroacupuncture as an adjunctive therapy for motor dysfunction in acute stroke survivors: a systematic review and meta-analyses. BMJ Open 2018;8:e017153. doi:10.1136/ bmjopen-2017-017153

- Prepublication history and additional material for this paper are available online. To view these files, please visit the journal online (http://dx.doi. org/10.1136/bmjopen-2017017153).

Received 4 April 2017

Revised 27 November 2017

Accepted 29 November 2017

Check for updates

${ }^{1}$ Graduate School, Guangzhou University of Chinese Medicine, Guangzhou, China

${ }^{2}$ Department of Neurology, Foshan Hospital of Traditional Chinese Medicine, Guangzhou University of Chinese Medicine, Foshan, China

${ }^{3}$ Department of Rehabilitation, Guangdong Provincial Hospital of Chinese Medicine, Guangzhou, China

${ }^{4}$ Key Unit of Methodology in Clinical Research, Guangdong Provincial Hospital of Chinese Medicine, Guangzhou, China ${ }^{5}$ National Centre for Design Measurement and Evaluation in Clinical Research, Guangzhou University of Chinese Medicine, Guangzhou, Guangdong, China

Correspondence to

Dr Zehuai Wen;

wenzh@gzucm.edu.cn

\section{ABSTRACT}

Objectives To assess the effectiveness and safety of electroacupuncture $(E A)$ combined with rehabilitation therapy (RT) and/or conventional drugs (CD) for improving poststroke motor dysfunction (PSMD).

Design Systematic review and meta-analysis.

Methods The China National Knowledge Infrastructure, Chinese Biological Medicine Database, Chinese Scientific Journal Database, Cochrane Library, Medline and Embase were electronically searched from inception to December 2016. The methodological quality of the included trials was assessed using the Cochrane risk of bias assessment tool. Statistical analyses were performed by RevMan V.5.3 and Stata SE V.11.0.

Results Nineteen trials with 1434 participants were included for qualitative synthesis and meta-analysis. The methodological quality of the included trials was generally poor. The meta-analysis indicated that the EA group might be benefiting more than the non-EA group in terms of the changes in the Fugl-Meyer Assessment Scale (FMA) (weighted mean difference (WMD): 10.79, 95\% Cl 6.39 to $15.20, \mathrm{P}<0.001$ ), FMA for lower extremity (WMD: 5.16, $95 \% \mathrm{Cl} 3.78$ to $6.54, \mathrm{P}<0.001)$ and activities of daily living (standardised mean difference: $1.37,95 \% \mathrm{Cl} 0.79$ to 1.96 , $\mathrm{P}<0.001)$. However, there was no difference between $\mathrm{EA}$ and non-EA groups in terms of the effective rate (relative risk: $1.13,95 \% \mathrm{Cl} 1.00$ to $1.27, \mathrm{P}=0.050$ ). Moreover, there were not any reports of side effects due to EA combined with $\mathrm{RT}$ and/or CD in the included trials.

Conclusions This review provides new evidence for the effectiveness and safety of EA combined with RT and/or CD for PSMD. However, the results should be interpreted cautiously because of methodological weakness and publication bias. Further clinical trials with a rigorous design and large sample sizes are warranted.

PROSPERO registration number CRD42016037597.

\section{INTRODUCTION}

Stroke is part of the world's leading causes of death and disability, ${ }^{12}$ causing heavy burdens to patients' families, communities and healthcare systems. ${ }^{3}$ Motor dysfunction is a frequent and widely recognised complication that often follows stroke. Approximately $85 \%$ of patients
Strengths and limitations of this study

- This systematic review with a comprehensive search of three English and three Chinese databases up to December 2016 was focused especially on assessing the adjunctive effects of electroacupuncture (EA) for motor dysfunction in acute stroke survivors within 14 days.

- Although the included trials in this review have methodological weakness, meta-regression analyses to explain the potential influence of the duration of treatment were performed, and sensitivity analyses with different risk of bias showed that the results were robust.

- Built on the low quality of included trials, we anticipate considerable difficulties in identifying the effectiveness of EA for motor dysfunction in acute stroke.

with stroke suffer from haemiparesis immediately after their stroke, and between $55 \%$ and $75 \%$ of stroke survivors may experience incomplete recovery with lingering motor dysfunction. ${ }^{4}$ Poststroke motor dysfunction (PSMD), which has a negative impact on the independence in functional activities, can reduce quality of life and limit activities of daily living (ADL). Therefore, effective treatment of PSMD is necessary to promote neurological function recovery and to alleviate the social and familial burdens of stroke.

Motor function recovery after stroke requires multidisciplinary treatment team and involves various approaches, such as conventional drugs $(\mathrm{CD})$, rehabilitation therapy (RT) and nursing care. RT plays an important role in comprehensive stroke rehabilitation programme aimed at recovering the function so as to reduce disabilities. Previous studies have demonstrated that neurological deficits due to stroke can benefit from RT. ${ }^{5}$ However, the effects of current RT for motor dysfunction caused by stroke are not optimal. ${ }^{6}$ Over 
the last decade, an increasing number of researchers have focused on alternative therapies for stroke rehabilitation, such as acupuncture.

In recent years, acupuncture, as one of the best known complementary medicines, has also been increasingly applied in China and other regions of the world. ${ }^{7}$ Electroacupuncture (EA), derived from the integration of traditional acupuncture and modern electrical stimulation, is another kind of acupuncture. EA is generally accepted because it is a relatively straightforward, safe and cheap therapy, compared with other conventional therapies. ${ }^{8}$ Additionally, EA has become more and more widely used in clinical practice because of its repeatability and standardisation of frequency, intensity and duration. ${ }^{910}$ After the needles are inserted into the acupuncture points, the electrodes are attached to the pairs of needles, and then a small electric current, usually with a pulse frequency of $1-100 \mathrm{~Hz}$ and pulse amplitude of 2-3 $\mathrm{mA}$, is passed through the needles into the subject for 15-60 min. ${ }^{11}{ }^{12}$ The efficacy of EA with strongly continued stimulation to treat peripheral facial paralysis is preferable to manual acupuncture. ${ }^{12}$ Furthermore, EA has been commonly recommended for use in clinic and research on acupuncture in China and in other countries. ${ }^{10}{ }^{13} \mathrm{EA}$ may improve functional recovery after stroke by inhibiting cell apoptosis, regulating the miR-9-mediated nuclear factor kappa-light-chain-enhancer of activated B cells downstream pathway and the miR-181b/paired immunoglobulin-like receptor B/ras homolog family member A/ growth-associated protein 43 axis, and dynamically maintaining the balance of matrix metallopeptidase- 9 and tissue inhibitor of metalloproteinase-1. ${ }^{14-16}$

A systematic review had suggested that EA was helpful in the treatment of acute ischaemic stroke, but its search time was up to June 2013 and was not focused on PSMD. ${ }^{17}$ Another newly published systematic review had revealed that EA had the potential to reduce spasticity within 180 days post stroke. ${ }^{18}$ However, these systematic reviews did not focus specifically on the effects of EA as an adjunctive therapy for motor dysfunction of acute stroke (within 14 days of onset ${ }^{19}$ ). Up to now, no clear evidence has been found that EA is more effective than non-EA in improving motor dysfunction within 14 days after stroke. Therefore, this study was conducted to assess the effectiveness and safety of EA combined with RT for PSMD in acute period, and to provide the best available evidence of clinical practice. The systematic review was registered on PROSPERO (https://www.crd.york.ac.uk/PROSPERO/) (trial registration number: CRD42016037597).

\section{METHODS}

\section{Types of studies}

We included all randomised controlled trials (RCTs) evaluating the effectiveness and safety of EA combined with RT for PSMD. The comparators or controls in the trials were any other therapy modalities. Also, we only included trials with outcomes measuring changes in motor function. All eligible trials published in Chinese or English were included, regardless of publication status.

\section{Types of participants}

We considered trials that included patients in the acute stage being the onset of their first stroke, with motor dysfunction measured by validated instruments or by a decrease in the level of movement activity. Patients were to be more than 18 years old and from any ethnicity. Stroke diagnosis had to meet the WHO criteria or the corresponding diagnostic criteria adopted in China, ${ }^{20-23}$ and had to be confirmed by CT or MRI. Trials involving participants with subarachnoid haemorrhages or cerebrovascular tumours, as well as those in which patients did not have acute stroke (within 14 days of onset ${ }^{19}$ ), were excluded.

\section{Types of interventions}

Patients in the experimental groups of the included trials had been treated with EA combined with RT and/or CD, at any frequency, intensity or duration. Patients in the control group of the trials had been treated by other therapies such as CD, RT and sham acupuncture, or had no treatments. However, trials that did not provide a detailed description or explanation of intervention, or those that compared different acupoint prescriptions or acupuncture types, were excluded.

\section{Primary outcome assessments}

The primary outcome for the systematic review was motor function. There are many types of motor function scales, including but not limited to the Fugl-Meyer Assessment Scale (FMA) ${ }^{24}$ the modified Rankin Scale, ${ }^{25}$ the Motor Assessment Scale ${ }^{26}$ and Brunnstrom Stages. ${ }^{27}$

\section{Secondary outcome assessments}

The secondary outcomes included measures of ADL, such as the Barthel Index (BI), ${ }^{28}$ the Functional Independence Measure (FIM), ${ }^{29}$ and the response or effective rate (ER). The ER was a standard of therapeutic effect recommended by the Fourth National Cerebrovascular Diseases Conference in China. The ER classified disability of stroke into five grades: cure (the scores of functional deficit were decreased up to $91 \%-100 \%$ ), significant improvement (the scores of functional deficit were decreased at $46 \%-90 \%$ ), improvement (the scores of functional deficit were decreased at 18\%-45\%), no improvement (the scores of functional deficit were decreased less than $18 \%$ ) and deterioration (the scores of functional deficit were increased over $18 \%) .{ }^{30}$ Safety assessments involved adverse events reporting and evaluation of causality. Adverse events as reported in the included trials were also recorded. The time of the outcome assessments was at the end of the intervention phase.

\section{Electronic searches}

We electronically searched databases from their respective inceptions up to December 2016. Databases included the China National Knowledge Infrastructure, 
Chinese Biological Medicine Database, Chinese Scientific Journal Database (VIP), Cochrane Library, Medline and Embase. We combined the PubMed search with the Cochrane highly sensitive search strategy for identifying randomised trials, and adapted this strategy to search the other databases.

We also searched other resources to identify potentially relevant trials. For example, we screened the reference lists of included trials and contacted the trial authors. The detailed search steps are illustrated in online supplementary appendix 1 .

\section{Data extraction and quality assessment}

Two review authors (JZ and MZ) independently scanned the titles and abstracts of the articles derived from the search and kept all potentially relevant articles. Then, they retrieved the full texts of these articles, and another two authors ( $\mathrm{ZH}$ and RP) independently examined them to confirm that the trials met the inclusion criteria. We also recorded the reasons for exclusion of trials. If the same trial had more than one report, we only kept one originally published version. If necessary, we acquired additional information from trial authors by email or telephone. Moreover, we discussed any disagreements to decide whether a trial should be included or excluded, and if necessary we consulted with another author (ZW).

Two authors (JZ and MZ) independently extracted information from the included trials. The information was entered into an Excel-formatted table (JZ) and the accuracy of the information entered was also checked (RP). The information extracted was as follows: trial design (eg, sample size, randomisation method, blinding method), participants (eg, gender, age), the details of intervention, outcomes (primary and secondary outcomes), adverse events, the name of the author, publication year and so on. Trial selection details are shown in a flow chart that complied with the Preferred Reporting Items for Systematic Reviews and Meta-Analyses (figure 1).

\section{Risk of bias assessment in the included studies}

Two review authors (JZ and RP) independently used the risk of bias assessment tool in the Cochrane Handbook for Systematic Reviews of Interventions ${ }^{31}$ to assess the methodological quality of each included trial. The specific domains were assessed as follows: random sequence generation, allocation sequence concealment, blinding,

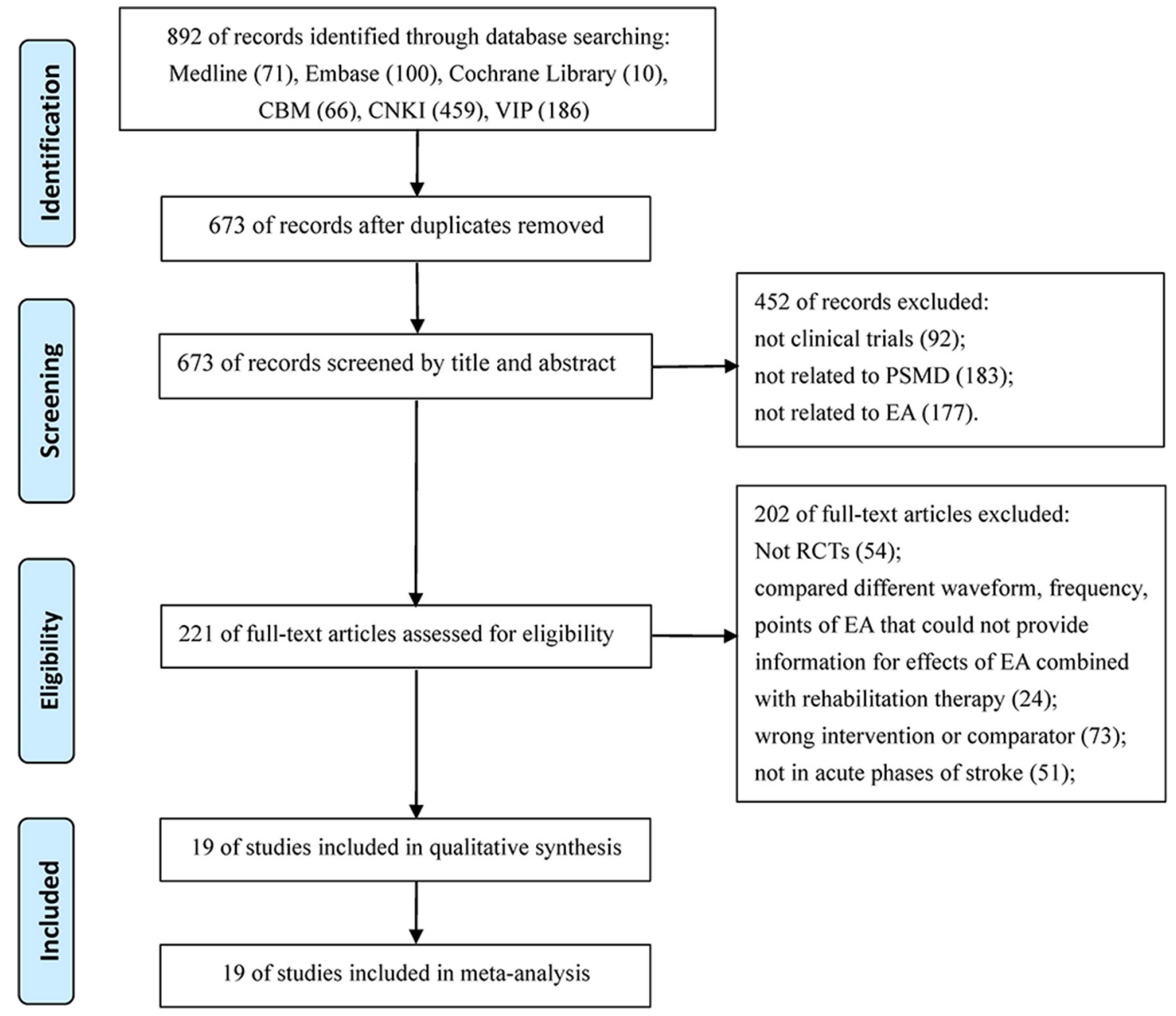

Figure 1 Study flow diagram. CBM, Chinese Biological Medicine Database; CNKI, China National Knowledge Infrastructure; EA, electroacupuncture; PSMD, poststroke motor dysfunction; RCT, randomised controlled trials; VIP, Chinese Scientific Journal Database. 
incomplete outcome data, selective outcome reporting and other sources of bias. We graded the risk of bias for each domain as low risk of bias, high risk of bias or unclear. We settled quality assessment disagreements by discussion with a third reviewer (ZW).

\section{Data analysis}

We performed all statistical analyses using RevMan V.5.3 (The Nordic Cochrane Centre, The Cochrane Collaboration 2014) and Stata SE V.11.0. For continuous data, we calculated the weighted mean difference (WMD) with corresponding $95 \%$ CI. If the outcomes were measured by different scales in the included trials, we calculated the standardised mean difference (SMD) with 95\% CI instead of WMD. For dichotomous data, we calculated the relative risk (RR) with $95 \%$ CI. We tested clinical and statistical heterogeneity among trials by comparing the characteristics of the trials, and used the $\mathrm{I}^{2}$ statistics to test heterogeneity. If heterogeneity was not significant, we chose a fixed-effects model to pool the data; otherwise, we used a random-effects model after considering clinical homogeneity. When heterogeneity was substantial, we examined trials for potential explanations, or else conducted a qualitative summary rather than a meta-analysis.

A meta-regression analysis was used to explain the potential trial-level covariates such as the duration of treatment. Subgroup analyses were carried out as follows: EA and RT plus CD compared with RT plus CD, and EA plus RT compared with RT alone. Sensitivity analysis was performed to explore the robustness of our analysis, excluding studies from the overall analysis of high risk of bias due to lack of allocation concealment and blinding of assessors for the primary outcome. If the number of included trials was greater than 10, funnel plots and Egger's test were employed for publication bias analysis.

\section{RESULTS}

\section{Trial description}

We initially identified 892 relevant articles according to the search strategy, while 219 duplicates from different databases were excluded. In total, 19 trials met the eligibility criteria after being screened by title, abstract or full texts, and were included in the meta-analysis (figure 1). We did not find additional trials for this review by examining the reference lists of included trials. All trials were published between 2004 and 2016. One trial ${ }^{32}$ was published in English, while the others were all published in Chinese. A total of 1434 participants were enrolled in these trials. All trials were conducted in China. Sixteen trials ${ }^{32-47}$ compared EA plus CD and RT with CD plus RT. Three trials $^{48-50}$ gave EA and RT to the experimental groups, while the control groups only received RT. CD was used according to the Chinese national guidelines, including general supportive care and specialised care such as antiplatelet agents, anticoagulants, fibrinogen-depleting agents, volume expansion and vasodilators. Because the patients enrolled in the trials were all within 14 days after stroke, CD was used similarly in each included trial. The characteristics of the trials included in this review are shown in table 1 .

\section{Assessing risk of bias in the included trials}

In general, the methodological quality of the included trials was poor. In random sequence generation, seven trials ${ }^{32} 343538444850$ used proper generation methods with a low risk of bias, and the random number sequences were produced by either a random number table, computer software or drawing lots. One trial used an incorrect sequence generation method. ${ }^{49}$ Eleven trials ${ }^{33} 363739-4345-47$ did not describe the randomisation procedure clearly. Two trials ${ }^{32}$ used concealed envelopes, and the other trials did not report allocation concealment. Two trials $^{32} 34$ reported that outcome assessors were blind to group allocation. Two trials ${ }^{32} 34$ mentioned that investigators were unknown for allocation. One trial ${ }^{32}$ reported dropouts and conducted intention-to-treat analyses. In other sources of bias, 11 trials ${ }^{36} 3739-414345-4850$ had a high risk because of inadequate statistical methods. These trials had not described the specific steps and methods of statistical analysis. The results of the assessments are presented in figure 2.

\section{Primary outcomes}

\section{Fugl-Meyer Assessment Scale}

The primary outcome, FMA score, was mentioned in 13 trials with 1010 patients. ${ }^{32-41} 48-50$ The effect of EA on FMA between the EA and non-EA groups was evaluated by a random-effects model, owing to significant heterogeneity. A meta-regression analysis was used to explain the potential covariates. The treatment duration was included as a potential covariable in the meta-regression model because the duration was from 2 to 12 weeks. However, there were no significant differences in treatment duration in the meta-regression model (adjusted $\mathrm{R}^{2}: 0.124, \mathrm{t}=-1.57$, $\mathrm{P}=0.144)$. The FMA score in the EA group increased more than those in the non-EA group, and there was a significant difference (WMD 10.79, 95\% CI 6.39 to 15.20 , $\mathrm{P}<0.001$ ) (figure 3 ).

\section{FMA for upper extremity}

One trial ${ }^{47}$ with 98 participants used the FMA for upper extremity to evaluate the function of the upper extremity, and the difference between the EA group and the non-EA group was obvious $(\mathrm{P}<0.050)$.

\section{FMA for lower extremity}

The function of the lower extremity was assessed by FMA for lower extremity (FMA-L) in four trials ${ }^{42-45}$ with 234 participants. The effect on FMA-L was analysed using a fixed-effects model, and there was a significant difference between the EA group and the non-EA group in the FMA-L (WMD 5.16, 95\% CI 3.78 to $6.54, \mathrm{P}<0.001$ ) (table 2). A meta-regression analysis was also carried out to explain the potential impact of treatment duration, and there was no significance in treatment duration in 


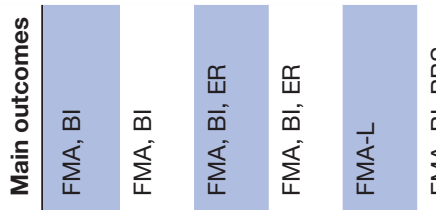

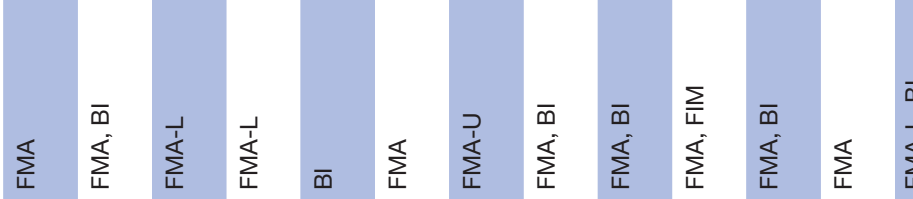

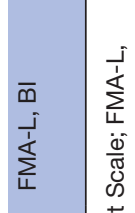

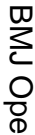

$\overrightarrow{\vec{F}}$

흔

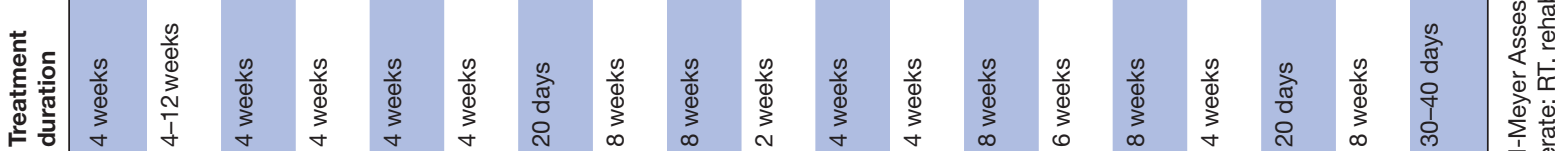

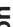

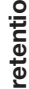

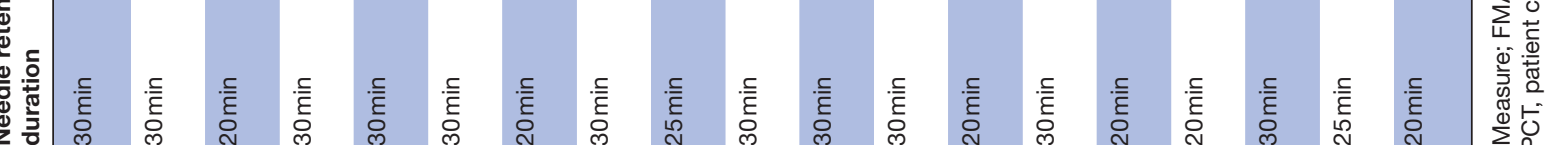

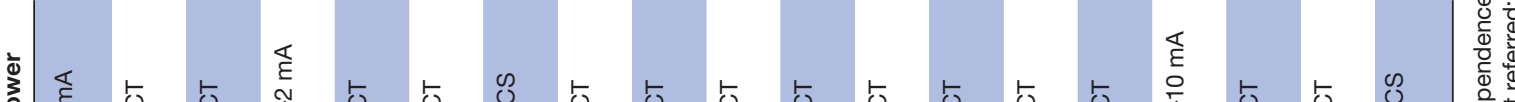

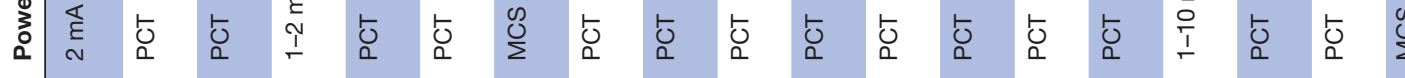

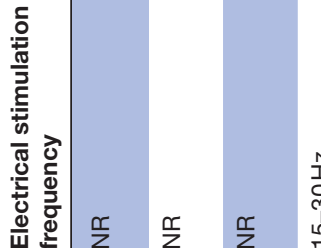

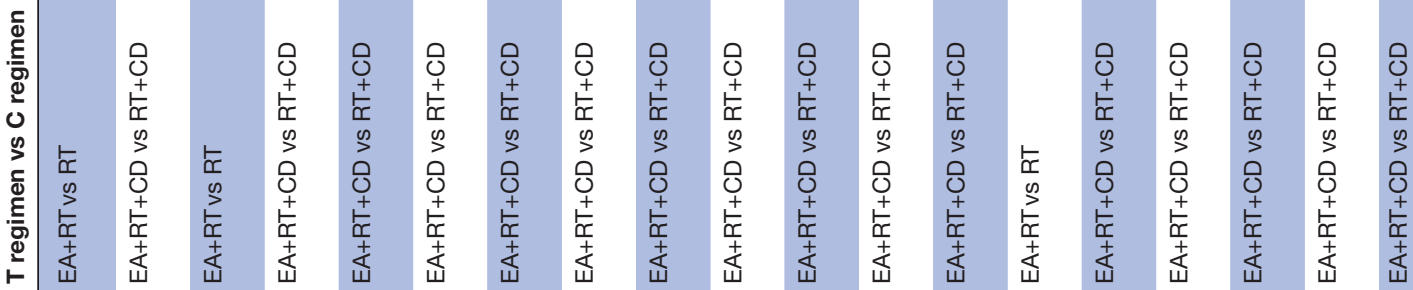




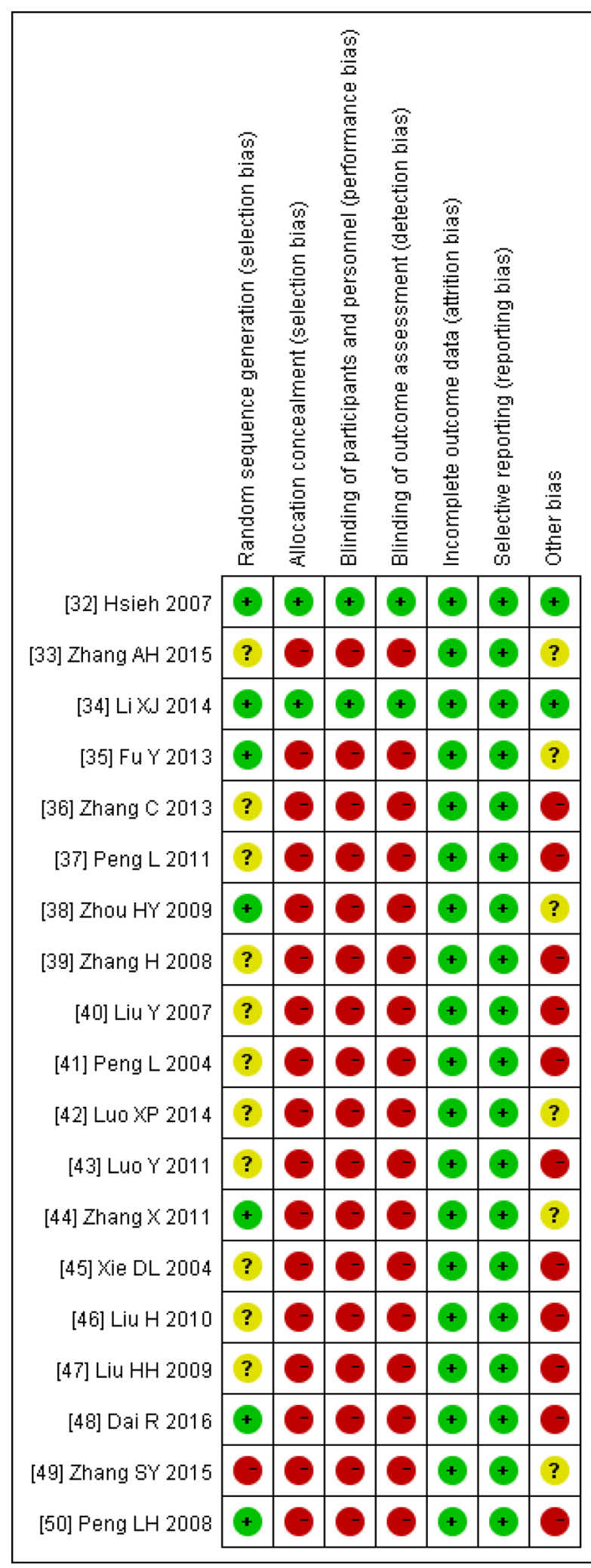

Figure 2 Risk of bias assessments of included studies.

the meta-regression model (adjusted $\mathrm{R}^{2}:-0.198, \mathrm{t}=-0.86$, $\mathrm{P}=0.482$ ).

\section{Secondary outcomes}

Activities of daily living

The effect of EA on ADL was analysed using a random-effects model, due to significant heterogeneity in 12 trials ${ }^{32-35373940454648-50}$ with 970 participants. We calculated the SMD with $95 \% \mathrm{CI}$ as the outcomes were measured by different scales (FIM and BI) in the included trials. The improvement of ADL in the EA group was better than that in the non-EA group (SMD 1.37, 95\% CI 0.79 to 1.96, $\mathrm{P}<0.001$ ) (figure 4).

\section{Response or ER}

Two trials ${ }^{3449}$ with a total of 171 participants showed that there was no significant difference in ER between EA and non-EA groups (RR 1.13, 95\% CI 1.00 to $1.27, \mathrm{P}=0.050$; fixed-effects model) (table 2).

Berg Balance Scale

Berg Balance Scale (BBS) was assessed in one trial ${ }^{35}$ with 120 participants. The improvement of BBS in the EA group was preferable to that in the non-EA group $(\mathrm{P}<0.050)$.

\section{Safety assessment}

None of the included trials mentioned adverse events due to both EA combined with RT and/or CD and RT and/or CD alone.

\section{Subgroup analysis}

EA plus RT and CD versus RT plus CD

Ten trials ${ }^{32-41}$ used FMA to measure the motor function of 796 participants with PSMD. A random-effects model was used to analyse the effect on FMA and ADL in this subgroup analysis due to significant heterogeneity. There was a significant difference between EA combined with RT and CD versus RT plus CD (WMD 8.03, 95\% CI 5.17 to 10.90, $\mathrm{P}<0.001$ ) (figure 3). Nine trials ${ }^{32-35} 3739404546$ used $\mathrm{BI}$ or FIM to measure the ADL of 756 patients following PSMD. EA plus RT and CD for the improvement of ADL was better than that of RT plus CD (SMD 1.29, 95\% CI 0.55 to $2.02, \mathrm{P}<0.001$ ) (figure 4 ).

\section{EA plus RT versus RT alone}

Three trials ${ }^{4-50}$ with 214 participants applied FMA to compare the effectiveness of EA plus RT against RT alone. Meta-analyses with a random-effects model were performed to evaluate the effect on FMA and ADL in this subgroup analysis owing to statistical heterogeneity. There was a significant difference in these three trials (WMD 20.90, 95\% CI 18.61 to $23.19, \mathrm{P}<0.001$ ) (figure 3 ). In the comparison of EA plus RT versus RT alone in the three trials, the difference in ADL was obvious (SMD $1.63,95 \%$ CI 1.01 to $2.25, \mathrm{P}<0.001$ ) (figure 4 ).

\section{Sensitivity analysis}

We used the method of removing item by item to test the stability of meta-analysis, and the results showed that there had been no noticeable change on any of the outcomes. The difference between the random-effects and fixed-effects models may have influenced the outcomes. Therefore, we used different statistical models to pool the data for the FMA, FMA-L, ADL and ER. No observable change in any of the outcomes was found (table 2). 


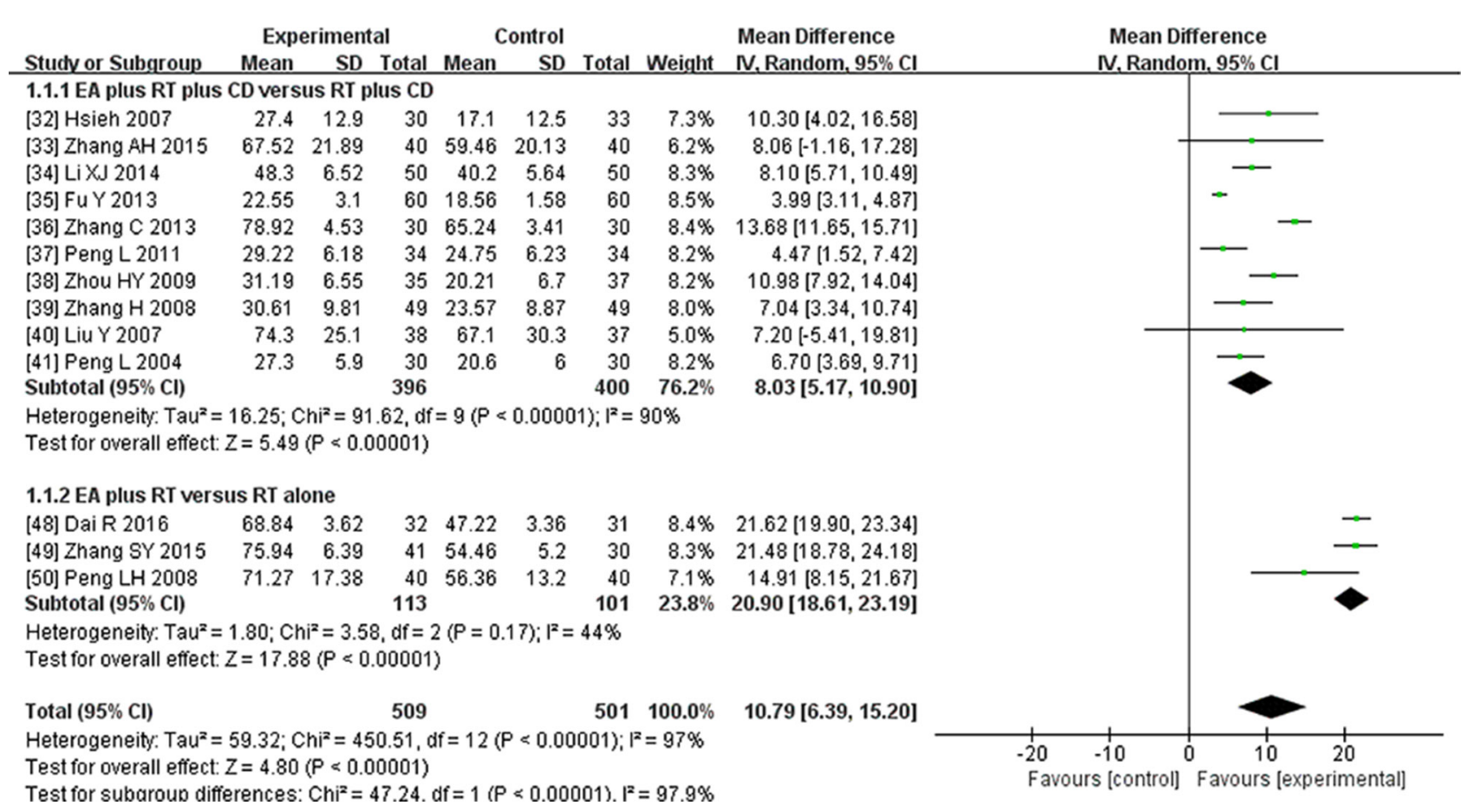

Figure 3 Forest plot and meta-analysis of Fugl-Meyer Assessment Scale. CD, conventional drugs; EA, electroacupuncture; RT, rehabilitation therapy.

Furthermore, sensitivity analysis was carried out to explore the robustness of our analysis, excluding trials from the overall analysis of high risk of bias due to lack of adequate sequence generation, allocation concealment and blinding of assessors for primary outcomes (table 2). The effects on FMA, ADL and ER were robust with random-effects and fixed-effects models with adequate sequence generation, with the exception of the comparison of EA plus RT versus RT alone and the trial subgroups (table 2).

\section{Publication bias}

Thirteen trials ${ }^{32-41} 48-50$ and 12 trials $^{32-35} 373940454648-50$ respectively showed a difference in FMA and ADL between the EA and the non-EA groups. Egger's tests showed that there were publication biases for the included trials of FMA $(\mathrm{t}=5.21, \mathrm{P}<0.001)$ or ADL $(\mathrm{t}=3.61, \mathrm{P}=0.005)$. The funnel plots showed that some trials did not lie inside the $95 \%$ CI and these were asymmetric. This may indicate potential publication bias (figure 5 and figure 6 ).

\section{DISCUSSION}

This systematic review included 19 RCTs with 1434 participants comparing the effectiveness and safety of EA therapy and non-EA therapy. The meta-analysis of four RCTs with 234 patients showed that adjunctive EA was better in improving the motor function of the lower extremity. One RCT with 98 patients demonstrated that added EA was beneficial for upper extremity motor control. The meta-analysis of 13 RCTs with 1010 patients indicated that adjunctive EA had greater advantage in the recovery of overall motor function, and the pooled results of 12 RCTs with 970 patients revealed that adjunctive EA was beneficial in the improvement of ADL. There was no any difference in the ER between EA and non-EA. However, it should be noted that the review included add-on designed trials of EA plus RT and/or $\mathrm{CD}$, which suggests that EA is a complementary therapy for PSMD. Meanwhile, there were insufficient data to assess the safety of EA plus RT, EA plus CD, and EA plus both $\mathrm{CD}$ and RT because none of the included trials reported adverse events. Considering the pooled effects on FMA and FMA-L with significant heterogeneity, a meta-regression analysis was conducted to explain the impact of treatment duration as a covariable. The result showed that the treatment duration was not significant in the meta-regression model. This means the heterogeneity could not be explained by the trial level's treatment duration.

One should be cautious when interpreting the results of the review due to some limitations. In this review, most of the included trials had small sample sizes. Sixty-three per cent of the included trials used a high risk of bias method or did not describe the generation of a random sequence. Eighty-nine per cent of the trials did not report allocation concealment and had inadequate blinding of outcome assessments. Fifty-eight per cent of the trials used inadequately designed statistical methods or did not fully describe the statistical methods. Only two trials ${ }^{32} 34$ were well-designed to assess the effect of EA combined with RT for PSMD. Additionally, all trials included in this review were conducted in China and most were published in Chinese, which likely lead to a potential bias and therefore limit their representativeness. 


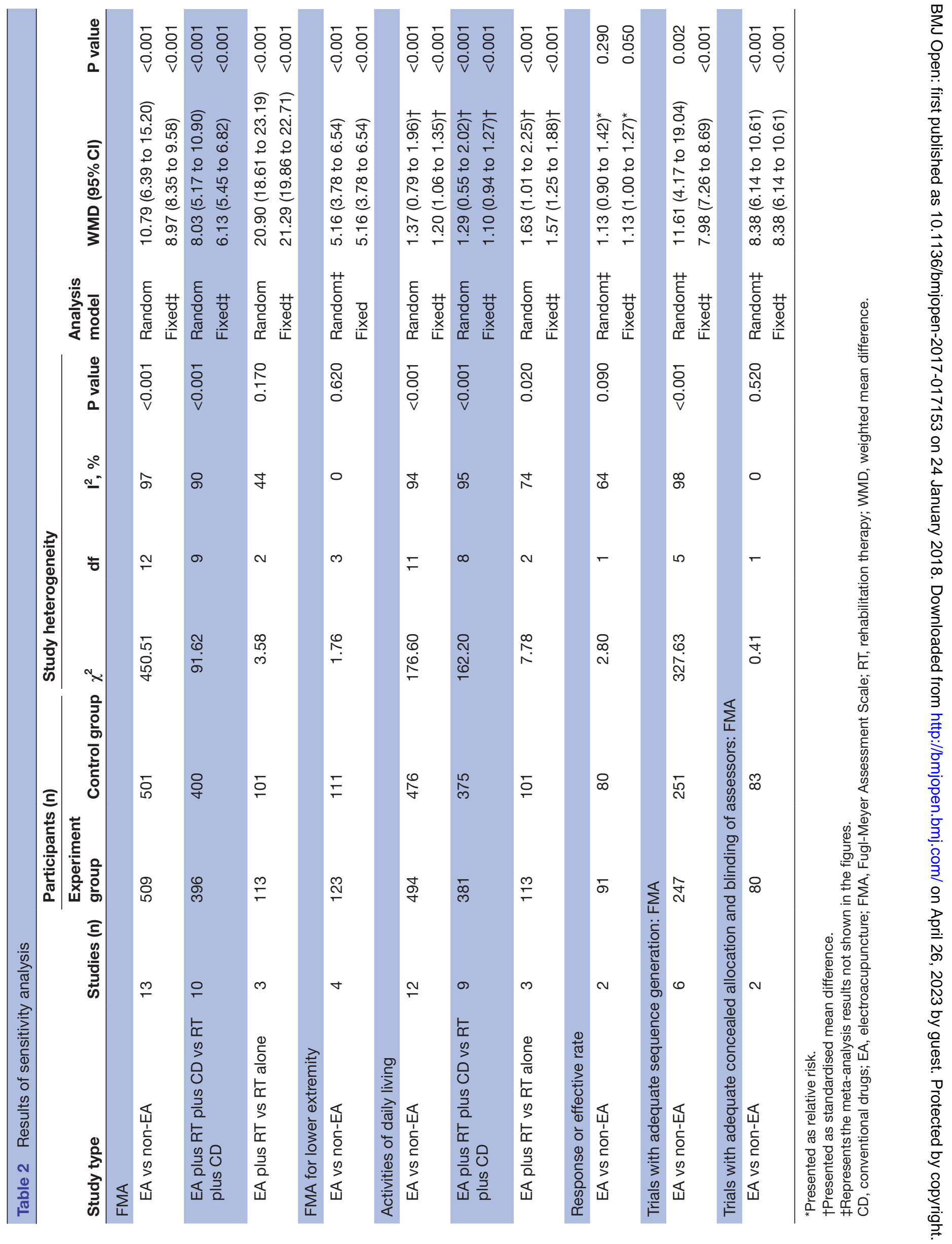




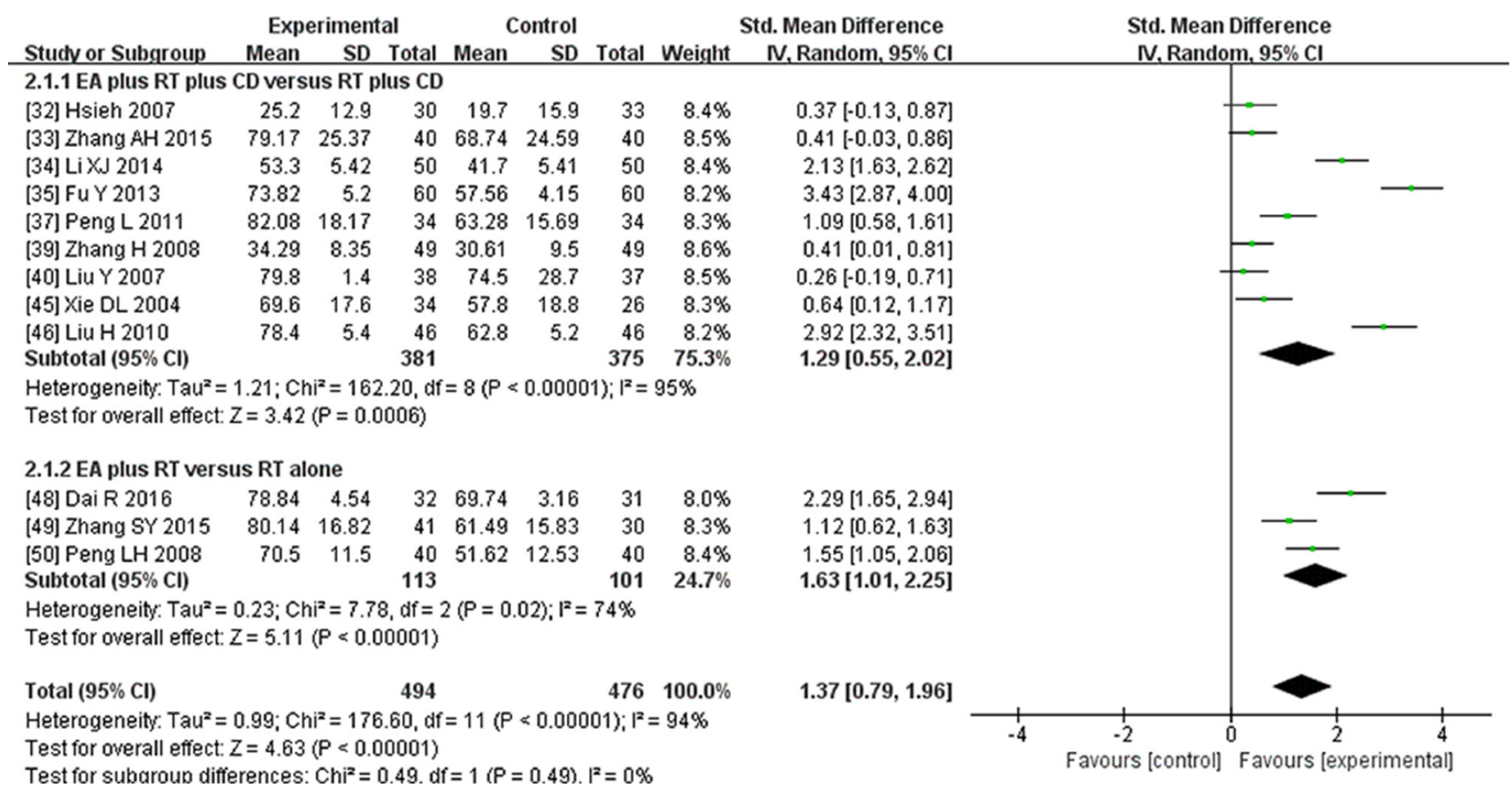

Figure 4 Forest plot and meta-analysis of activities of daily living. CD, conventional drugs; EA, electroacupuncture; RT, rehabilitation therapy.

Two previous systematic reviews were reported to assess the effects of EA on the treatment of ischaemic stroke. ${ }^{1718}$ However, there were noticeable differences in the study characteristics of participants, comparison and outcomes, and both reviews did not focus on evaluating EA as an adjunctive therapy for PSMD of acute stage within 14 days of onset. We still need to gain a clear evidence for PSMD in this critical stage, which will influence the prognosis of stroke.
Most of the included trials had methodological defects, and the funnel plots of FMA and ADL suggested a potential publication bias. These issues potentially lead to low quality of evidence, over-reporting of positive results and under-reporting of adverse events. Also, the use of diverse $\mathrm{RT}$ as an add-on basis in the included trials makes it difficult to pool data using a fixed-effect model to interpret the clinical significance of EA. Therefore, the potential benefits of EA as an adjunctive therapy for PSMD evident

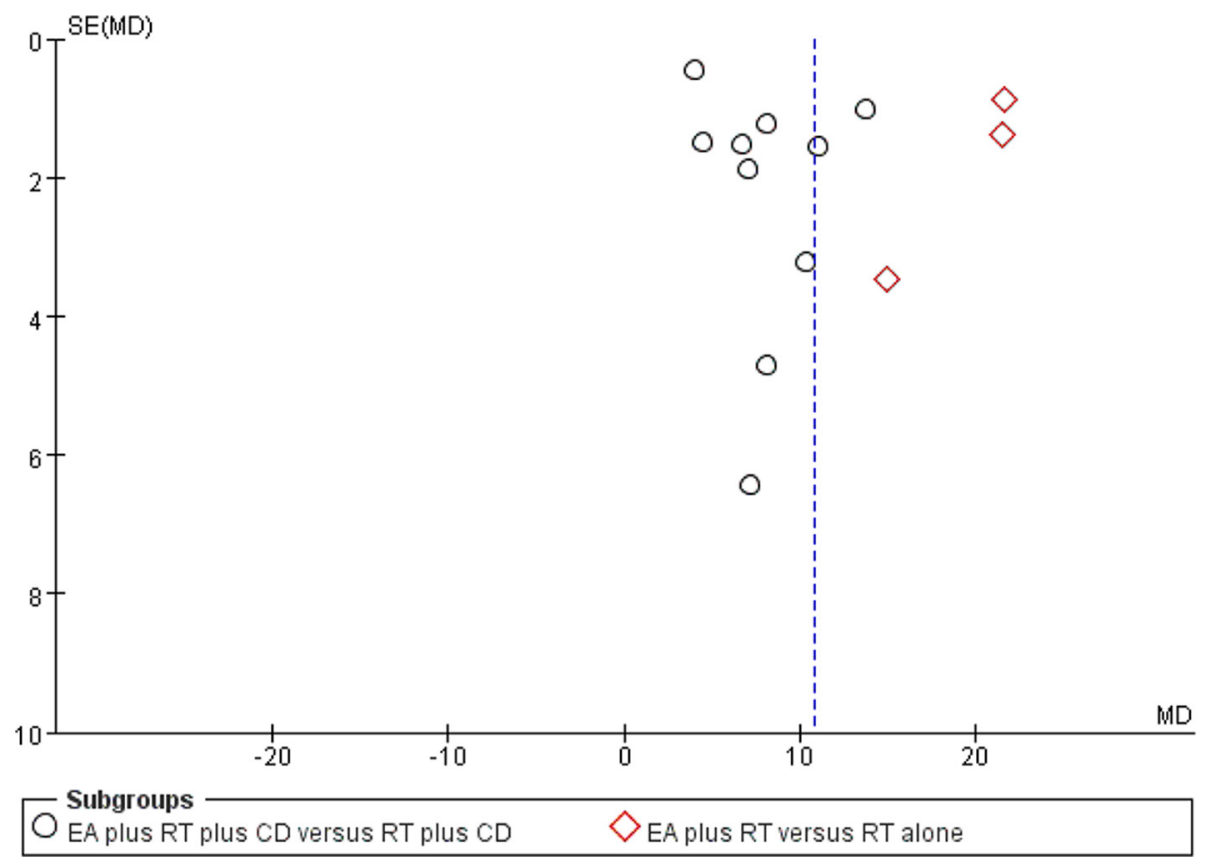

Figure 5 Funnel plots illustrating meta-analysis of Fugl-Meyer Assessment Scale. CD, conventional drugs; EA, electroacupuncture; MD, mean difference; RT, rehabilitation therapy. 


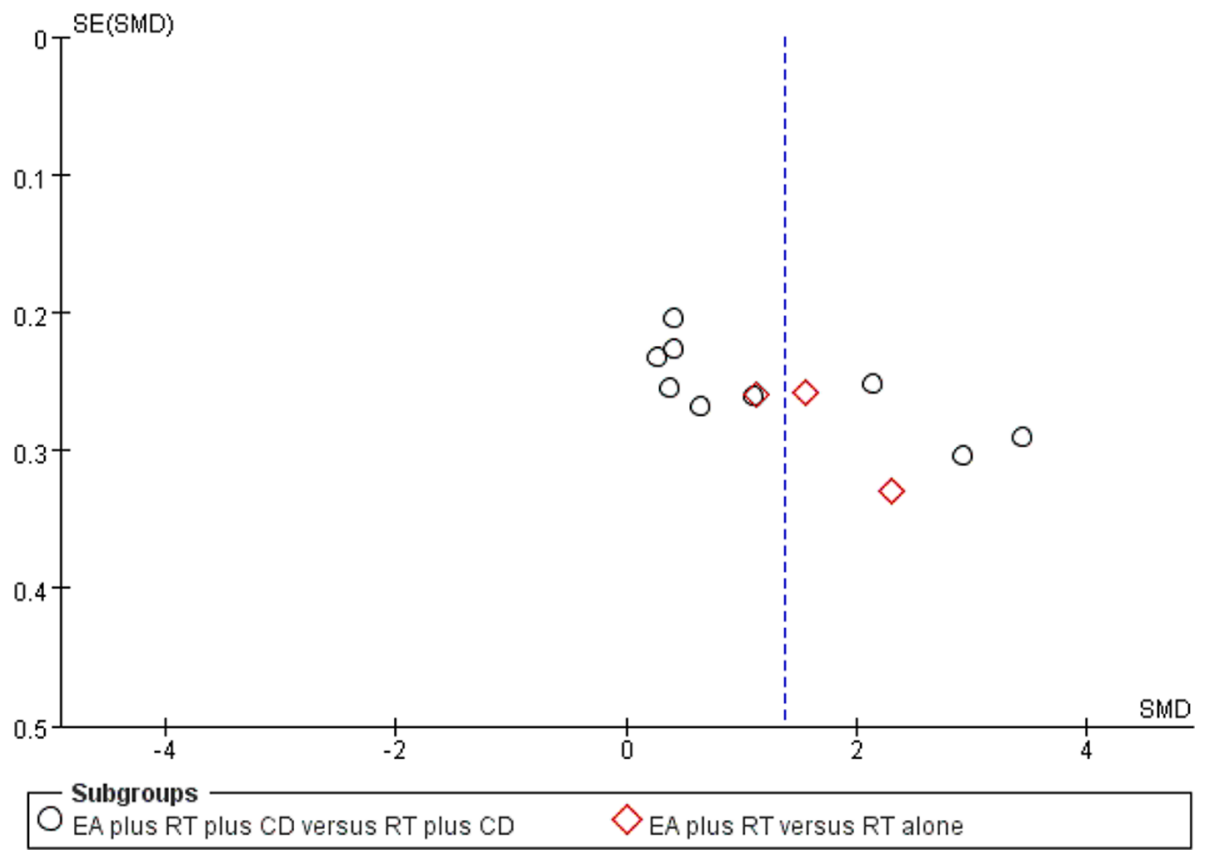

Figure 6 Funnel plots illustrating meta-analysis of activities of daily living. CD, conventional drugs; EA, electroacupuncture; RT, rehabilitation therapy; SMD, standardised mean difference.

in this review need to be further appraised through well-designed, large-scale, multicentre RCTs.

\section{CONCLUSIONS}

EA as a complementary therapy seems to have clinical benefits in terms of improving the function of extremities, ADL and balance function. However, these apparent benefits require further evaluation through well-designed multicentre trials with large sample sizes. The safety of EA combined with RT and/or CD is still uncertain.

Acknowledgements We would like to thank Mr Ben Ross from the University of Chicago, engaging in research of sociology and urbanism in China, for his assistance in language editing of this paper.

Contributors $\mathrm{JZ}$ and $\mathrm{ZW}$ were responsible for conception and design of this systematic review. The manuscript was drafted by JZ, and revised by ZW and FT. The search strategies were designed by ZW and JZ. The electronic search was conducted by JZ, MZ and JD. ZH manually searched key journals. JZ and MZ extracted the data. The risk of bias was assessed by JZ and RP, independently. JZ and $Z W$ analysed and interpreted the data. ZW arbitrated any disagreements in the process of systematic review. All authors approved this manuscript.

Funding This work received a grant from the National Key Technology R\&D Program for the 12th Five-Year Plan of Ministry of Science and Technology, China (no 2013BAI02B10).

Competing interests None declared.

Patient consent Not required.

Provenance and peer review Not commissioned; externally peer reviewed.

Data sharing statement No additional data are available.

Open Access This is an Open Access article distributed in accordance with the Creative Commons Attribution Non Commercial (CC BY-NC 4.0) license, which permits others to distribute, remix, adapt, build upon this work non-commercially, and license their derivative works on different terms, provided the original work is properly cited and the use is non-commercial. See: http://creativecommons.org/ licenses/by-nc/4.0/
(C) Article author(s) (or their employer(s) unless otherwise stated in the text of the article) 2018. All rights reserved. No commercial use is permitted unless otherwise expressly granted.

\section{REFERENCES}

1. Mozaffarian D, Benjamin EJ, Go AS, As G, et al. Heart disease and stroke statistics-2016 Update: A Report From the American Heart Association. Circulation 2016;133:e38-e360.

2. Sacco RL, Kasner SE, Broderick JP, et al. An updated definition of stroke for the 21st century: a statement for healthcare professionals from the American Heart Association/American Stroke Association. Stroke 2013;44:2064-89.

3. Liu L, Wang D, Wong KS, et al. Stroke and stroke care in China: huge burden, significant workload, and a national priority. Stroke 2011:42:3651-4.

4. Nichols-Larsen DS, Clark PC, Zeringue A, et al. Factors influencing stroke survivors' quality of life during subacute recovery. Stroke 2005;36:1480-4.

5. Murphy TH, Corbett D. Plasticity during stroke recovery: from synapse to behaviour. Nat Rev Neurosci 2009;10:861-72.

6. Lefebvre S, Dricot L, Laloux P, et al. Neural substrates underlying stimulation-enhanced motor skill learning after stroke. Brain 2015;138(Pt 1):149-63.

7. Anon. NIH Consensus Conference. Acupuncture. JAMA 1998;280:1518-24.

8. Barnes PM, Bloom B, Nahin RL. Complementary and alternative medicine use among adults and children: United States, 2007. Natl Health Stat Report 2008:1-23.

9. Zhou F, Guo J, Cheng J, et al. Electroacupuncture increased cerebral blood flow and reduced ischemic brain injury: dependence on stimulation intensity and frequency. J Appl Physiol 2011;111:1877-87.

10. Wei JJ, Yang WT, Yin SB, et al. The quality of reporting of randomized controlled trials of electroacupuncture for stroke. BMC Complement Altern Med 2016;16:512.

11. Chen CY, Ke MD, Kuo CD, et al. The influence of electro-acupuncture stimulation to female constipation patients. Am J Chin Med 2013;41:301-13.

12. Mayor D. Electroacupuncture: an introduction and its use for peripheral facial paralysis. J Chin Med 2007;84:1-17.

13. Napadow V, Ahn A, Longhurst J, et al. The status and future of acupuncture mechanism research. $J$ Altern Complement Med $2008 ; 14: 861-9$ 
14. Liu W, Wang $X$, Zheng $Y$, et al. Electroacupuncture inhibits inflammatory injury by targeting the miR-9-mediated NF- $\mathrm{kB}$ signaling pathway following ischemic stroke. Mol Med Rep 2016;13:1618-26.

15. Deng B, Bai F, Zhou H, et al. Electroacupuncture enhances rehabilitation through miR-181b targeting PirB after ischemic stroke. Sci Rep 2016;6:38997.

16. Ma R, Yuan B, Du J, et al. Electroacupuncture alleviates nerve injury after cerebra ischemia in rats through inhibiting cell apoptosis and changing the balance of MMP-9/TIMP-1 expression. Neurosci Lett 2016;633:158-64.

17. Liu AJ, Li JH, Li HQ, et al. Electroacupuncture for acute ischemic stroke: a meta-analysis of randomized controlled trials. Am J Chin Med 2015;43:1541-66.

18. Cai Y, Zhang CS, Liu S, et al. Electroacupuncture for poststroke spasticity: a systematic review and meta-analysis. Arch Phys Med Rehabil 2017;98:2578-89.

19. Yang W, Shi Z, Yang HQ, et al. Mailuoning for acute ischaemic stroke. Cochrane Database Syst Rev 2015;1:CD007028.

20. Stroke--1989. Recommendations on stroke prevention, diagnosis, and therapy. Report of the WHO Task Force on Stroke and other Cerebrovascular Disorders. Stroke 1989;20:1407-31.

21. Wang $Y$. The criteria of traditional Chinese medicine for diagnosis and evaluation of stroke. China J Trad Chinese Med 1986;1:56-7.

22. Ministry of Health of the People's Republic of China. Clinical research guidelines for new traditional Chinese drug, 1993.

23. Chen QT. Classification, diagnostic criteria and evaluation of neurological impairment for stroke patients. Chinese $J$ Neurol 1996;29:376-83.

24. Fugl-Meyer AR, Jaasko L, Leyman I, et al. The poststroke hemiplegic patient: I. a method for evaluation of physical performance. Scand $J$ Rebabil Med 1975;7:13-31.

25. Bonita R, Beaglehole R. Recovery of motor function after stroke. Stroke 1988;19:1497-500.

26. Carr JH, Shepherd RB, Nordholm L, et al. Investigation of a new motor assessment scale for stroke patients. Phys Ther 1985;65:175-80.

27. Brunnstrom S. Motor testing procedures in hemiplegia: based on sequential recovery stages. Phys Ther 1966;46:357-75.

28. Mahoney FI, Barthel DW. Functional evaluation: the barthel index. Md State Med J 1965;14:61-5.

29. Hamilton BB, Laughlin JA, Fiedler RC, et al. Interrater reliability of the 7-level functional independence measure (FIM). Scand J Rehabil Med 1994;26:115-9.

30. The Forth National Cerebrovascular Diseases Conference. Classification, diagnostic criteria and evaluation of neurological impairment for stroke patients (1995). Chinese J Neurol 1996;29:381-3.

31. In: Higgins JPT, Green S, eds. Cochrane handbook for systematic reviews of interventions version 5.1.0 [updated March 2011]. 2011: The Cochrane Collaboration. www.cochrane-handbook.org

32. Hsieh RL, Wang LY, Lee WC. Additional therapeutic effects of electroacupuncture in conjunction with conventional rehabilitation for patients with first-ever ischaemic stroke. J Rehabil Med 2007;39:205-11.

33. Zhang AH. Clinical observation of electroacupuncture combined with modern rehabilitation on cerebrovascular hemiplegia. Asia-Pacific Trad Med 2015;11:92-3.
34. Li XJ, Ch H, Wang XY, et al. Clinical study on electroacupuncture at scalp acupoints plus kinesitherapy for post-stroke hemiplegia. Shanghai J Acu-mox 2014;33:979-82.

35. Fu Y, Liu JX, Wang DL, et al. Influence of electric-acupuncture combined with limbs intensive training on motor function of hamiplegic limb in stroke patient. Chinese Nurs Res 2013;27:1743-5.

36. Zhang C, Liu J, Lin QH, et al. Effect of electrical acupuncture on the movement functions of the patients with acute cerebral infarction (ACl). J TCM Univ Hunan 2013;33:79-80.

37. Peng L, Luo J, Zhang L, et al. Effect of combined electroacupuncture and neural rehabilitation technique on limb function of patients with intracerebral hemorrhage. Mil Med J S Chin 2011;25:477-8.

38. Zhou HY, Wd J, Wang SS, et al. Effects of electro-acupuncture on muscle tension and motor function in flaccid paralytic limbs after stroke. Chin J Phys Med Rehabil 2009;31:401-3.

39. Zhang H, Li L. Effect of early electro-acupuncture on locomotion of hemiplegia patients after stroke. Chin J Rehabil Theory Pract 2008;14:824-5

40. Liu Y, Zou SJ. Effect of electroacupuncture on motor function of acute stroke patients received early rehabilitation. Chin J Rehabil Theory Pract 2007;13:969-70.

41. Peng L, Ly L, Peng R, et al. Electroacupuncture in combination with promote treatment technique for the recovery of ambulation in the patients with acute apoplexy. Chinese J Rehabil 2004;19:26-7.

42. Luo XP. Effect of electro-acupuncture combined with rehabilitation training on lower limb motor function in strok patients with hemiplegia. J Tradit Chin Med 2014;32:159-61.

43. Luo Y. Effect of electroacupuncture combined with neurodevelopmental therapy on walking function in patients with acute stroke. Chinese J Trauma Disabil Med 2011;19:129-30.

44. Zhang X, Tan F, Duan CZ, et al Effect of electro-needling at acupoints of the yangming meridian on the expression of PAC- 1 and lower limb functions In acute cerebral infarction patients. Chinese $J$ Integrat Trad Western Med.

45. Xie DL, Zhu LF, Liu HY, et al. The Effect of Electroacupuncture Plus Lower Limb Function Intensive Training on Lower Limb Function in Patients with Acute Cerebral Infarction. Shanghai J Acu-mox 2004;23:21-2.

46. Liu H. Clinical observation of early rehabilitation training combined with electroacupuncture on activity of daily living in patients with acute stroke. Zhongguo Zhong Xi Yi Jie He Za Zhi 2010;2:251-3.

47. Liu HH, Zhang H, Zhang XS, et al. Effect of Electro-acupuncture to Upper-limb Movement Function in Hemiplegia Patients with Stroke in Early Stage. Journal of Liaoning University of Traditional Chinese Medicine 2009;11:157-8.

48. Dai R, Sun HM, Tc M, et al. Effects of electrical acupuncture combined with physical therapy on motor function of patients with acute stroke. Medicine and Pharmacy of Yunnan 2016;37:8-12.

49. Zhang SY, Peng BW, Liu SB, et al. Effect of electroacupuncture combined with rehabilitation training for 41 patients with stroke hemiplegia. J Hubei Univ Nationalit 2015;32:77-80.

50. Peng LH, Zeng QH, Rh M. Influence of motor function and activities of daily life in patients with hemiparalysis caused by cerebral stroke with neural stem electro acupuncture combined with rehabilitation. Modern Hospital 2008;8:80-1. 